\title{
Hemangioma hepático gigante
}

\section{Giant hepatic hemangioma}

Veronica Argüello-Ramírez ${ }^{*}$, Julio C. Gómez-Penagos², Martha Castro-Ibarra², Jesús R. Soto-Pérez², Abraham Cañavera-Constantino ${ }^{3}$ y M. de Guadalupe Gómez-Pérez ${ }^{1}$

${ }^{1}$ Servicio de Resonancia Magnética, Hospital Ángeles del Pedregal; 2Servicio de Radiología e Imagen, Hospital Ángeles Lindavista; ${ }^{3}$ Servicio de Patología, Hospital Ángeles Lindavista. Ciudad de México, México

\section{RESUMEN}

El hemangioma hepático es el tumor benigno del hígado más frecuente. Suele ser de pequeño tamaño, único o múltiple, pero en ocasiones supera los $4 \mathrm{~cm}$, y en este caso se denomina hemangioma hepático gigante ${ }^{1}$. En general son asintomáticos y se detectan de forma casual, aunque a veces, como en nuestro caso, pueden provocar dolor o molestias abdominales. Su diagnóstico se basa en estudios imagenológicos y se confirma con el estudio de histopatología de la biopsia o de la resección quirúrgica.

Palabras clave: Hemangioma hepático. Dolor abdominal. Necrosis hepática submasiva. Hematoma subcapsular.

\section{ABSTRACT}

Hepatic hemangioma is the most common benign tumor of the liver; It is usually small, single or multiple, but sometimes exceeds $4 \mathrm{~cm}$, called hepatic giant hemangioma ${ }^{1}$. In general, they are asymptomatic and are detected by chance, although sometimes, as in our case, they can cause pain or abdominal discomfort. Its diagnosis is based on imaging studies and is confirmed with the histopathology study in biopsy or surgical resection.

Key words: Hepatic hemangioma. Abdominal pain. Submassive hepatic necrosis. Subcapsular hematoma. 


\section{INTRODUCCIÓN}

Los hemangiomas hepáticos son los tumores hepáticos benignos más comunes, con una prevalencia del 7\% en autopsias y del 1 al 20\% en la población en general ${ }^{1-4}$. Son más comunes en las mujeres, probablemente como resultado de la influencia de las hormonas sexuales femeninas en su crecimiento ${ }^{5}$. Los hemangiomas hepáticos gigantes, definidos como aquellos con un tamaño mayor de $4 \mathrm{~cm}$, representan el 10\% de todos los hemangiomas hepáticos.

Por lo general son asintomáticos y su hallazgo es incidental ${ }^{6}$. Se han reportado síntomas hasta en el $40 \%$ de los pacientes con hemangiomas hepáticos gigantes en algunas series, principalmente dolor abdominal, pero también síntomas relacionados con un efecto de masa, como saciedad temprana, náuseas, vómito, colestasis o hasta tos ${ }^{7-10}$. Las manifestaciones menos comunes incluyen ascitis quilosa, hemoperitoneo debido a una rotura espontánea y síndrome de Kasabach-Merritt, en el cual el hemangioma hepático se asocia con trombocitopenia y coagulación intravascular.

El aspecto ecográfico de estas lesiones clásicamente se ha descrito como lesiones bien delimitadas, homogéneas e hiperecoicas debido a la interfase de las paredes de los senos cavernosos y la sangre en su interior. Otras características que se pueden encontrar, sobre todo en las lesiones de mayor tamaño, son una zona central no homogénea, con áreas hipoecoicas de aspecto granular, o también descrito como encaje, borde ecogénico y un contorno festoneado. También es común encontrar en los hemangiomas gigantes focos centrales hipoecoicos que se corresponden con cicatrices fibrosas. Es raro observar calcificaciones.

En cuanto a la vascularidad, presentan un flujo extremadamente lento que no suele detectarse con ninguna de las modalidades de Doppler.

Esta heterogeneidad en las lesiones, sobre todo las de mayor tamaño, hace necesaria su confirmación con los estudios contrastados dinámicos, generalmente tomografía computarizada trifásica, en la que clásicamente se mostrará un realce centrípeto muy característico, donde en la fase arterial se presentará un realce periférico $\mathrm{y}$, posteriormente, en las siguientes adquisiciones, un progresivo realce con tendencia a la homogeneidad. El papel de la resonancia magnética es caracterizar con detalle las lesiones hepáticas. Su sensibilidad y especificidad es del 98\%. Estos hemangiomas aparecerán hipointensos en T1 e hiperintensos en T2. La resonancia magnética, además, permite el uso de secuencias especiales y fases dinámicas con gadolinio, y la ventaja de ser un método multiplanar.

El diagnóstico diferencial incluye angiomatosis, metástasis, quistes hemáticos, peliosis hepática y carcinoma hepatocelular, entre otros.

Las opciones de tratamiento del hemangioma hepático, incluyendo el hemangioma hepático gigante, son la observación, enucleación, resección hepática y embolización arterial transcatéter. Recientemente, la ablación por radiofrecuencia progresiva se ha utilizado para el tratamiento de los hemangiomas hepáticos gigantes, $\mathrm{y}$ ha mostrado buenos resultados ${ }^{11}$. 


\section{CASO CLÍNICO}

Paciente de sexo femenino, de 22 años de edad, que refiere como antecedente de importancia la presencia de un tumor hepático desde hace 2 años sin manejo alguno.

Niega uso de hormonales. Gesta 0. Fecha de la última regla: 4 de marzo de 2019.

Inicia el padecimiento actual en el mes de marzo de 2019. Se produce posterior a la ingesta de alimentos, con dolor abdominal de intensidad aguda localizado en el epigastrio, de tipo transfictivo, sin irradiaciones, y en una ocasión asociado a vómito de contenido gastrobiliar.

A la exploración física, neurológicamente íntegra, Glasgow de 15, bien hidratada, sin compromiso cardiorrespiratorio, abdomen blando, depresible, doloroso a la palpación en el epigastrio y el hipocondrio derecho, sin datos de irritación peritoneal, extremidades integras sin alteraciones. Signos vitales con frecuencia cardíaca de 76 latidos por minuto, frecuencia respiratoria de 22 respiraciones por minuto, tensión arterial de $110 / 60 \mathrm{~mm} / \mathrm{Hg}$, temperatura de $36.5^{\circ}$ y saturación $98 \%$.

Se realizan los que se describen a continuación (Tabla 1).

En los estudios de gabinete se le realiza un ultrasonido abdominal en el que se observa hepatomegalia secundaria a la presencia de lesión ecogénica, ovoidea, de bordes difusos, la cual presenta vascularidad periférica al Doppler color localizada en los segmentos VII y VIII, con dimensiones aproximadas de 12.9 $\times 15.2 \mathrm{~cm}$ en sus ejes mayores, así como

\begin{tabular}{|c|c|}
\hline Leucocitos & 11.9 miles/ul \\
\hline Hemoglobina & $13.8 \mathrm{~g} / \mathrm{dl}$ \\
\hline Hematocrito & $45 \%$ \\
\hline Plaquetas & 410 miles $/ \mu \mathrm{l}$ \\
\hline Glucosa & $103 \mathrm{mg} / \mathrm{dl}$ \\
\hline Fósforo & $5.6 \mathrm{mg} / \mathrm{dl}$ \\
\hline Potasio & $5.1 \mathrm{mEq} / \mathrm{l}$ \\
\hline Cloro & $105 \mathrm{mEq} / \mathrm{l}$ \\
\hline Bilirrubina total & $0.78 \mathrm{mg} / \mathrm{dl}$ \\
\hline Transaminasa glutámico-oxalacética & $38 \mathrm{U} / \mathrm{l}$ \\
\hline Alanino-aminotransferasa & $24 \mathrm{U} / \mathrm{I}$ \\
\hline Deshidrogenasa láctica & $195 \mathrm{U} / \mathrm{I}$ \\
\hline Amilasa & $98 \mathrm{U} / \mathrm{l}$ \\
\hline Lipasa & $37 \mathrm{U} / \mathrm{l}$ \\
\hline
\end{tabular}

imagen de morfología semilunar de localización subcapsular ecogénica, con dimensiones aproximadas de $12.9 \times 4.9 \mathrm{~cm}$. A considerar como primera posibilidad hemangioma hepático gigante asociado a hematoma subscapular (Figs. 1 A-B).

Posteriormente se decidió complementar el estudio con tomografía contrastada trifásica de abdomen, en la que se identificó la lesión antes descrita, que ocupa casi la totalidad del lóbulo derecho, con predominio de los segmentos VII y VIII. Hipodensa en fase simple, mostró realce de periférico a central (centrípeto), con zonas hipodensas centrales en relación con necrosis, con dimensiones aproximadas de $13.9 \times 11.3 \times 16.9 \mathrm{~cm}$ en sus ejes mayores, asociada a un hematoma subcapsular con un volumen aproximado de 589 cc y hemoperitoneo, el cual se extiende al hueco pélvico secundario a la ruptura de este (Figs. 2 A-C). 

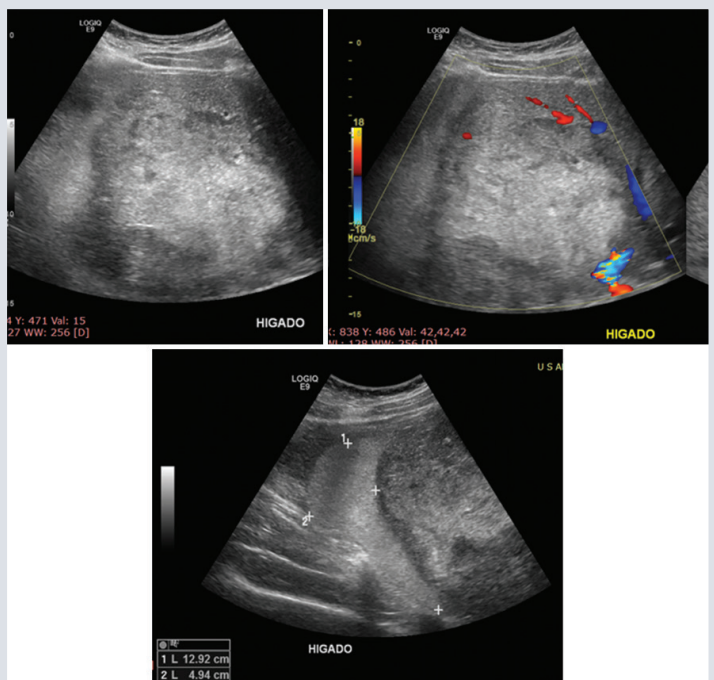

Figura 1. A-B: ecografía en plano transverso en donde se observa en segmentos VII y VII hepáticos lesión ecogénica, ovoidea, de bordes difusos que a la aplicación de Doppler color, se aprecia con vascularidad periférica. C: ecografia en plano transversal donde se observa imagen ecogénica de morfología semilunar de localización subcapsular en relación a hematoma.

Se realiza resonancia magnética (Figs. 3 A y B), en donde se observa la lesión antes descrita, de intensidad heterogénea, de predominio hipointensa en T1 e hiperintensa en T2, con áreas hipointensas centrales secundarias a las zonas de necrosis. No es posible realizar estudio dinámico debido a que la paciente presenta inestabilidad hemodinámica, sin embargo, con las secuencias adquiridas fue posible corroborar el diagnóstico de hemangioma hepático gigante.

En las pruebas de laboratorio de control se observa un descenso de la hemoglobina de hasta $6.8 \mathrm{~g}$, por lo que se decide llevar a cabo el procedimiento quirúrgico. Se realizó una lobectomía derecha y se envió muestras a patología, donde los datos que se reportan son compatibles con hemangioma hepático asociado a zonas de necrosis $y$ hemorragia.
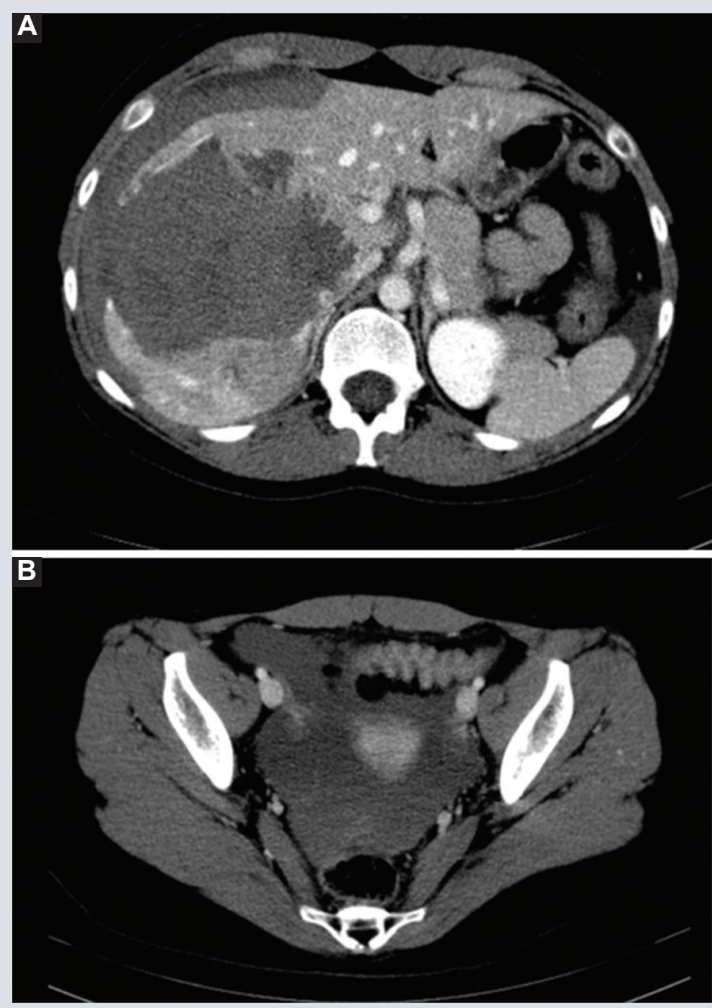

Figura 2. Tomografía contrastada en plano axial, donde se observa una lesión hepática de predominio hacia los segmentos VII y VIII con zonas de necrosis central, la cual presenta realce centrípeto, asociada a un hematoma subcapsular $\left({ }^{*}\right)$ (A). A nivel del hueco pélvico, se observa hemoperitoneo (B).

Reporte histopatológico:

- Necrosis hepática submasiva (afección estimada del 75\% de la muestra evaluada) asociada a infiltrado inflamatorio agudo de moderado a intenso y hemorragia extensa.

- Trombosis de vasos venosos de pequeño calibre.

- Fragmentos de coágulo hemático con escasas áreas de parénquima hepático y con zonas de necrosis y zonas con cambios degenerativos (Fig. 4).

- Descripción microscópica: los cortes histológicos teñidos con técnica de hematoxilina-eosina muestran el parénquima 

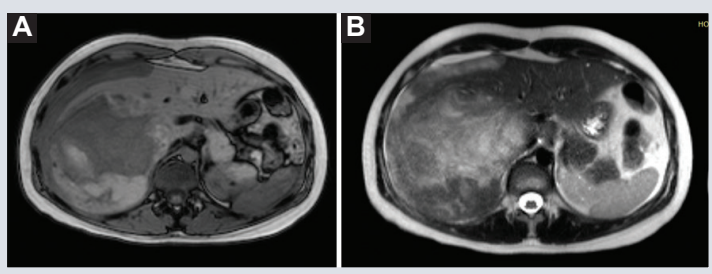

Figura 3. Resonancia magnética potenciada. A: T1 en plano axial que muestra una lesión heterogénea de predominio hipointenso con áreas hiperintensas en relación con un hemangioma hepático gigante asociado a hematoma subcapsular; B: T2 en plano axial que muestra una lesión hepática de predominio hiperintenso con áreas hiperintensas.

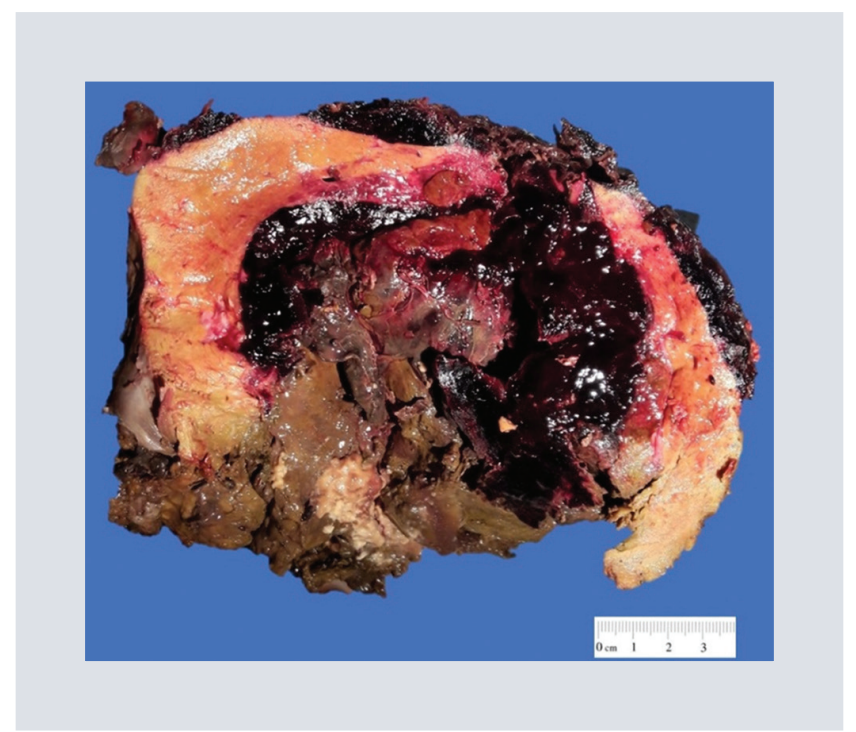

Figura 4. Fragmento de tejido hepático irregular que presenta zonas hemorrágicas sólidas y friables alternando con pequeñas bandas ahuladas y gris blanquecino.

hepático con extensas áreas con detritus celulares entremezclados con grupos de células inflamatorias, con predominio de neutrófilos, rodeadas por zonas con pérdida del detalle celular e incremento en la eosinofilia. Se identifican algunas venas de pequeño calibre con oclusión total y parcial de sus luces por láminas de eritrocitos alternando con láminas de fibrina $\mathrm{y}$

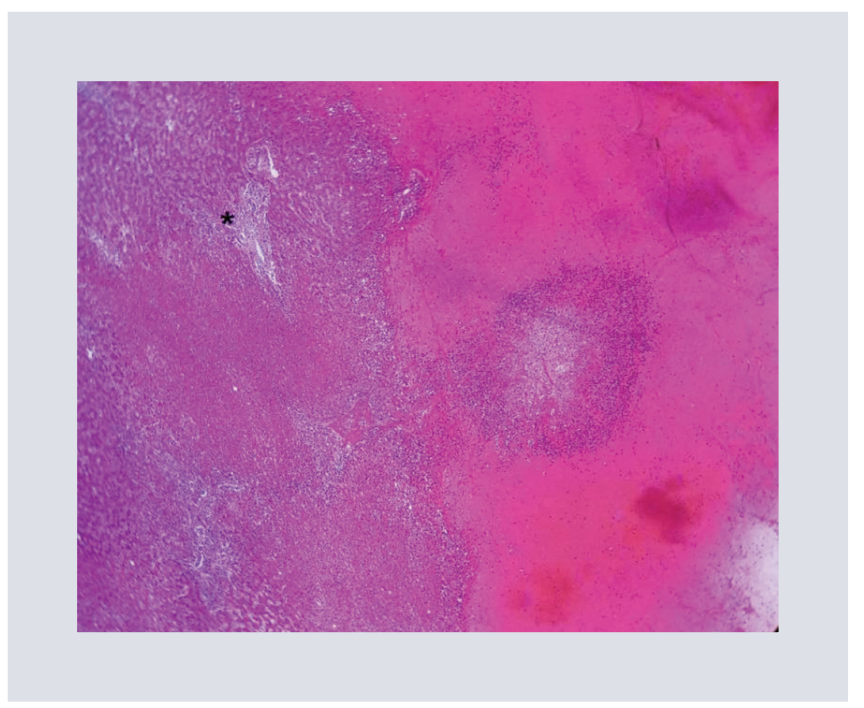

Figura 5. Fotomicrografía a bajo aumento $\times 40$ con tinción de hematoxilina-eosina, donde se observa necrosis centrolobulillar con preservación de los hepatocitos adyacentes a la zona alrededor de las triadas portales $(*)$.

células inflamatorias. Las áreas de parénquima hepático residual muestran cambios regenerativos acentuados con identificación nódulos y de zonas con proliferación colangiolar reactiva (Fig. 5)

\section{DISCUSIÓN}

Los hemangiomas hepáticos gigantes representan solo el 10\% de los hemangiomas hepáticos, miden más de $4 \mathrm{~cm}$, son más frecuentes en mujeres y la mayoría de ellos son asintomáticos. Cuando presentan síntomas, el dolor es la queja más frecuente.

Son lesiones benignas cuya causa no es completamente entendida. Se consideran malformaciones vasculares congénitas que crecen por ectasia y no por hiperplasia o hipertrofia. Se ha asociado su crecimiento a factores hormonales (estrógenos) con base en reportes de crecimiento durante el embarazo, sin embargo, 
no se han demostrado receptores de estrógenos en todos los hemangiomas y puede existir crecimiento en mujeres posmenopáusicas o usuarios de anticonceptivos orales.

Con la evolución de las técnicas de imagen, el diagnóstico se hace por lo general basándose en estudios radiológicos. Se empieza con la ecografía, sin embargo, por su tamaño es difícil establecer el diagnostico, ya que no presenta las características habituales, además de que en esta paciente presentaba zonas de necrosis debido al tiempo de evolución, por lo que fue necesario complementar con otros métodos de imagen, como fue tomografía trifásica y la resonancia magnética, lo cual nos permitió establecer el diagnóstico certero, además de observar otros hallazgos asociados que suelen presentarse en dichos hemangiomas como el hematoma subcapsular, el cual mostraba ruptura y condicionó hemoperitoneo, lo que asociado a disminución de la hemoglobina determinó la urgencia quirúrgica, y se realizó una lobectomía derecha, la cual al estudio histopatológico corroboro nuestro diagnóstico.

\section{BIBLIOGRAFÍA}

1. Bioulac-Sage P, Laumonier H, Laurent C, Blanc JF, Balabaud C. Benign and malignant vascular tumors of the liver in adults. Sem Liv Dis. 2008;28(3):302-14.

2. Uetama T, Yoshida H, Hirakata A, Yokoyama T, Maruyama H, Suzuki S, et al. A symptomatic giant hepatic hemangioma treated with hepatectomy. J Nippon Med Sch. 2011;78(1):34-9.

3. Klinger C, Stuckmann G, Dietrich CF, Berzigotti A, Horger MS, Debove I, et al. Contrast-enhanced imaging in hepatic epithelioid hemangioendothelioma: retrospective study of 10 patients. Z Gastroenterol. 2019;57(6):753-66.

4. Lerut J, Iesari S. Vascular tumours of the liver: a particular story. Transl Gastroenterol Hepatol. 2018;3:62.

5. Hamaloglu E, Altun H, Ozdemir A, Ozenc A. Giant liver hemangioma: Therapy by enucleation or liver resection. World J Surg. 2005;29:890-3.

6. Shimada K, Nakamoto Y, Isoda H, Saito H, Arizono S, Shibata T, et al. FDG PET for giant cavernous hemangioma: Important clue to differentiate from a malignant vascular tumor in the liver. Clin Nucl Med. 2010;35:924-6.

7. Bozkaya H, Cinar C, Besir FH, Parıldar M, Oran I. Minimally invasive treatment of giant hemangiomas of the liver: Embolisation with bleomycin. Cardiovasc Intervent Radiol. 2014;37:101-7.

8. Lazarus DL, Al-Busafi SA, Hilzenrat N. Chylous ascites secondary to giant liver hemangioma. Case Rep Gastroenterol. 2012; 6(2):4784-83.

9. Doklestic K, Stefanovic B, Karamarkovik A, Bumbasirevic V, Stefanovic B, Gregorić $\mathrm{P}$, et al. Spontaneous rupture of giant liver hemangioma: Case report. Srp Arh Celok Lek. 2013;95-9.

10. Rossi UG, Camisassi N, Pinna F, Rollandi GA. Spontaneous hepatic haemangioma rupture and hemoperitoneum: a double problem with a single stage interventional radiology solution. Clin Exp Emerg Med. 2019;6(2):169-72.

11. Dima-Cozma LC, Bitere OR, Pantazescu AN, Gologan E, Mitu F, Rădulescu D. Cavernous liver hemangioma complicated with spontaneous intratumoral hemorrhage: a case report and literature review. Rom J Morphol Embryol. 2018;59(2):557-61. 